\title{
О.А. ГОВОРУХИНА
}

\section{ДИАГНОСТИКА И ЛЕЧЕНИЕ БОЛЕЗНИ ГИРШПРУНГА У ДЕТЕЙ НА СОВРЕМЕННОМ ЭТАПЕ}

\author{
ГУ «Республиканский научно-практический центр детской хирургии», г. Минск, \\ Республика Беларусь
}

В обзоре проведен анализ литературы, отражающей современные взгляды на болезнь Гиршпрунга у детей, а также современные методы диагностики и лечения заболевания.

Болезнь Гиршпрунга - достаточно частое заболевание в детской хирургии. Изучение литературы показало, что если вопросы патогенеза заболевания уже не считаются спорными, то внедрение новых методик обследования и хирургического лечения по-прежнему остается сложным и неоднозначным. Применение тех или иных методов диагностики и лечения определяется в основном возможностями и предпочтениями лечебных учреждений. В то же время функциональные результаты оперативного лечения оставляют желать лучшего. Причинами этого являются отсутствие четко определенных сроков проведения хирургической коррекции порока, а также нерешенные вопросы выбора метода оперативного лечения, подходящего для каждого конкретного ребенка.

Анализ литературных данных показал возможность решения этих проблем путем выработки рациональной программы обследования с учетом всех имеющихся осложнений и определения соответствующей тактики лечения.

Радикальное одноэтапное оперативное лечение болезни Гиршпрунга с использованием миниинвазивных методов приобретает все большее распространение. Современные хирургические методы лечения болезни Гиршпрунга у новорожденных и детей раннего возраста позволяют снизить смертность и улучшить результаты.

Ключевые слова: болезнь Гиршпрунга, аганглиоз, толстая кишка, энтероколит, трансанальное эндоректальное низведение, послеоперационные осложнения

The paper reviews recent studies that evaluate the present-day concept concerning Hirschsprung's disease in children as well as current diagnostic and treatment methods of this pathology.

Hirschsprung's disease is a relatively common disease in the pediatric surgery. The literature analysis has shown that if the pathogenesis of the disease is no longer considered controversial entity, the application of new methods of examination and surgical treatment still remains complex and ambiguous. The use of one or another method of diagnosis and treatment is thought to be determined mainly by the possibilities and preferences of medical establishment. At the same time, the functional results of surgical treatment leave much to be desired due to the absence of clearly defined terms for surgical correction of the defect, as well as the unresolved issues of choice of operative treatment method appropriated for each specific child.

The analysis of the literature data has shown the possibility of solving these problems by developing a rational program of examination, taking into account all existing complications and determining the appropriate treatment tactics.

Radical one-stage surgical treatment of Hirschsprung's disease with the use of minimally invasive methods is becoming more widespread. Current surgical methods for treating Hirschsprung's disease in infant and young children are essential in reducing morbidity and mortality associated with this disease.

Keywords: Hirschsprung's disease, agangliosis, colon, enterocolitis, transanal endorectal pull-through, postoperative complications, mortality

Novosti Khirurgii. 2017 Sep-Oct; Vol 25 (5): 510-517

Current Diagnosis and Treatment of Hirschsprung's Disease in Children

O.A. Govorukhina

\section{Введение}

Болезнь Гиршпрунга - одна из сложных проблем в детской хирургии. Значимые успехи были достигнуты в понимании ее этиологии в последние десятилетия, особенно с развитием молекулярной генетики и новых патоморфологических исследований.

Новый этап в совершенствовании методов диагностики и лечения болезни Гиршпрунга получил развитие не только благодаря успехам патофизиологии и раннему выявлению заболевания, но и благодаря развитию хирургической техники. Хирургическое лечение продвинулось от двух- или трехэтапных операций до первично-радикальной [1, 2, 3]. В последнее время радикальная коррекция болезни Гиршпрунга при помощи миниинвазивной техники стала все более популярной. У новорожденных и детей раннего возраста прогрессивные методы 
лечения болезни Гиршпрунга способствуют снижению смертности и улучшению результатов лечения. Анализ литературы по рассматриваемой теме позволит представить современное состояние вопроса.

\section{Этиология}

Клеточные и молекулярные нарушения в процессе развития энтеральной нервной системы и миграции нервных гребешковых клеток в развивающийся кишечник представляют собой первичную этиологию болезни Гиршпрунга. Нейробласты впервые появляются в формирующемся пищеводе после 5 недели гестации плода. Эти клетки мигрируют в краниокаудальном направлении в остальную развивающуюся кишку между 5 и 12 неделями гестации. Формы болезни Гиршпрунга различны из-за огромного количества возможных аномалий во время развития энтеральной нервной системы и различного времени, когда происходит остановка миграции нейробластов. Ранняя остановка миграции в развивающемся эмбрионе приводит к длинной зоне аганглиоза [2].

Другие факторы, такие как повреждение экстраклеточных матричных компонентов, аномалии нейротрофических факторов, молекулярные повреждения нервных клеток могут также рассматриваться как способствующие факторы развития болезни Гиршпрунга.

\section{Генетические факторы}

Увеличение риска рождения детей с болезнью Гиршпрунга среди родственников пациентов с данным заболеванием, преобладание по полу, сочетание с болезнью Гиршпрунга других пороков, синдромов и хромосомных нарушений доказывают наличие генетических факторов болезни Гиршпрунга. Генетические исследования выявили мутацию в 10 различных генах, способствуюших развитию болезни Гиршпрунга [4]. Самыми частыми среди них являются мутации в RET гене (7-35\% спорадических случаев), EDNRB гене (7\%) и END3 гене (менее 5\%) [3, $4,7]$. Более 20 различных мутаций было описано в RET protooncogene, некоторые изменения в этом гене сочетаются с определенными формами болезни Гиршпрунга [3, 5].

И даже если болезнь Гиршпрунга возникает как изолированная форма, она сочетается с врожденными пороками и сопутствует синдромам (таким, как трисомия 21, септальные дефекты сердца, врожденный центральный гиповентиляционный синдром, множественные эндокринные нарушения neoplasia type
2, нейрофиброматоз, Ваарденбурга синдром) в 5-32\% случаях [4]. Трисомия 21 (Даун-синдром) сочетается с болезнью Гиршпрунга в 7\% случаев. Сочетанные пороки наиболее часто встречаются в желудочно-кишечном тракте, затем в центральной нервной и мочеполовой системах $[4,5]$.

\section{Патофизиология}

В основе патогенеза болезни Гиршпрунга лежит функциональная непроходимость узкой части толстой кишки, затрудняющая образование перистальтических волн из-за отсутствия парасимпатических ганглионарных клеток. Несмотря на громадные исследования, причина тонического сужения аганглионарной кишки полностью не выяснена. Аганглиоз, холинергическая гипериннервация, нарушение обеспечения нервов нитрик-оксид синтетазой и нарушения интерстициальных клеток Кахаля в совокупности участвуют в патогенезе болезни Гиршпрунга, но полное понимание причинных факторов нарушений, видимых при болезни Гиршпрунга, не получено [2, 6].

\section{Классификация}

Распространение аганглиоза в проксимальном направлении от внутреннего анального сфинктера определяет классификацию: форма с короткой зоной аганглиоза - ректо-сигмоидное поражение (74-80\%); с длинной зоной (субтотальная) - аганглиоз распространяется до селезеночного угла или поперечно-ободочной кишки (12-22\%); тотальный аганглиоз толстой кишки - аганглиоз всей толстой и терминального участка подвздошной кишок (4-13\%). Выделяют также интестинальную и ультракороткую форму. Наиболее тяжелая, но редкая форма болезни Гиршпрунга - тотальный интестинальный аганглиоз кишечника с отсутствием ганглионарных клеток от дуоденум до ректум [7].

По клиническому течению заболевания выделяют неосложненную и осложненную формы болезни Гиршпрунга.

\section{Клинические проявления}

Частота болезни Гиршпрунга примерно 1 на 5000 рождений. Соотношение мальчиков к девочкам при ректосигмоидной форме $4: 1$, а при длинной зоне - 1:1-2:1.

С усилением настороженности и улучшении диагностики возраст диагностирования болезни Гиршпрунга существенно уменьшился 
в последнее время и в большинстве случаев диагностируется в периоде новорожденности. Позднее отхождение мекония, увеличение в объеме живота, рвота с примесью желчи и нарушения энтерального питания - наиболее частые симптомы [8].

Обычно новорожденные с болезнью Гиршпрунга - доношенные дети с поздним отхождением мекония. Почти у всех доношенных детей меконий отходит в первые 24-48 часов жизни. При болезни Гиршпрунга у 70-90\% новорожденных этого не происходит в данный период жизни. Дифференциальный диагноз нужно проводить со следующей патологией: тонкая кишка - кишечная атрезия, мальротация, заворот, мекониальная непроходимость при муковисцидозе; толстая кишка - meconium plug syndrome, аноректальные мальформации, small left colon syndrome; другие причины - наркотики, электролитные нарушения, гипотиреоидизм, сепсис, очень малый вес при рождении. Таким образом, болезнь Гиршпрунга должна быть заподозрена у всех детей с поздним отхождением стула в периоде новорожденности.

При осложненной форме болезни Гиршпрунга клиническая картина проявляется в виде низкой кишечной непроходимости или перфорации слепой кишки и перитонита в первые дни жизни ребенка, что затрудняет постановку диагноза в периоде новорожденности. Однако осложнения течения болезни Гиршпрунга могут наблюдаться и в более старшей возрастной группе пациентов, что связано с неизбежно возникающим при этом заболевании энтероколитом.

\section{Энтероколит при болезни Гиршпрунга}

Энтероколит - тяжелое осложнение болезни Гиршпрунга в до- и послеоперационных периодах. Это клиническое состояние с симптомами, включающими диарею, вздутие живота, повышение температуты тела, коликообразные боли в животе, интоксикацию, гиповолемию, жидкий стул, иногда с примесью крови.

Энтероколит может развиться в любом возрасте - от периода новорожденности до взрослого состояния - независимо от медикаментозного лечения или выполненного хирургического вмешательства. Рецидивирующий энтероколит может возникнуть даже при наличии выведенной стомы $[7,8]$

Несмотря на многочисленные исследования, полное понимание этиологии энтероколита еще отсутствует. Многочисленные теории были предложены, чтобы объяснить его возникновение, включая физическое расшире- ние проксимальной части кишки, изменение содержимого кишки, ротавирус, клостридию, повышение активности простагландина Е1, иммунологический дефект слизистой оболочки кишки и др. Некоторые гистологические и иммунологические исследования выявили локальный или системный иммунологический дефицит с нарушением функции лимфоцитов [9].

Частичная механическая непроходимость может запустить патогенез энтероколита по причине расширения проксимальной части кишки, что приведет к кишечному стазу, еще большему расширению кишки, ишемии слизистой и бактериальной инвазии. Однако, эта теория не объясняет возникновение энтероколита в дистальной от стомы части кишки, возникновение энтероколита в послеоперационном периоде или выявление энтероколита гистологически в аганглионарной кишке. Длина аганглионарного сегмента может рассматриваться как возможный фактор увеличения риска возникновения энтероколита. Несомненно, что большая длина аганглионарного сегмента вызывает более выраженную проксимальную обструкцию с большим давлением, увеличивающим бактериальный стаз и проксимальное расширение. Тем не менее, исследования показали отсутствие корреляции между длиной аганглионарного сегмента и частотой возникновения энтероколита.

Продукция муцина значительно снижена у пациентов с болезнью Гиршпрунга. Причем ганглионарный сегмент показывает сходные повреждения продукции слизи, как и в аганглионарном сегменте. Это указывает на ненормальный защитный слизистый барьер в кишке у пациентов с болезнью Гиршпрунга, даже в гистологически нормальной кишке. Снижение продукции слизи указывает на опасность бактериального повреждения слизистой оболочки и транслокации флоры.

Секреторный иммуноглобулин А обеспечивает основной иммунологический барьер в желудочно-кишечном тракте, устойчивость к бактериям и предотвращает бактериальную транслокацию через кишечную стенку. Несомненно, если наступил первый эпизод энтероколита, то он может нарушить процесс кишечного иммунитета выработкой соответствующих антител, быть причиной хронических изменений в слизистой оболочке кишки и повысить риск следующих эпизодов. Это может объяснить реальную возможность возникновения рецидива энтероколита после выведения стомы или успешной операции $[7,9]$.

Макрофаги играют важную роль в воспалении в мышечной оболочке. Увеличение 
количества и активности макрофагов при воспалении приводит к нарушению работы интерстициальных клеток Кахаля, отвечающих за ритмичность работы кишечника в участках кишки, в которых ганглии интактны. Эти нарушения перистальтики могут ухудшить стаз, бактериальный рост и вместе с повреждением продукции нормальной слизи усилить транслокацию [8].

Повреждения на уровне генов, запускающие механизмы развития энтероколита при болезни Гиршпрунга, подтверждаются тем, что, например, у детей с болезнью Дауна развивается энтероколит почти в $50 \%$ случаев, один и более эпизодов $[7,8]$. Присутствие других сопутствующих аномалий также вызывает увеличение частоты энтероколита.

При микробиологическом исследовании в большинстве случаев удается выделить Clostridium difficile $[7,8,9]$.

\section{Диагноз}

В связи с усилением настороженности педиатров и детских хирургов, возраст постановки диагноза болезни Гиршпрунга существенно снизился в последние годы и во многих случаях стал возможен еще в периоде новорожденности. Болезнь Гиршпрунга должна быть заподозрена у новорожденных с поздним отхождением мекония (в течение 24-48 часов), увеличением объема живота, рвотой с примесью желчи и нарушением энтерального питания. Наличие у новорожденного ребенка признаков энтероколита должно также вызывать подозрение на болезнь Гиршпрунга. Низкая кишечная непроходимость с сохранением анатомической целостности кишки, перфорации слепой, восходящей кишок или терминального отдела тонкой кишки, перитонит в первые дни жизни ребенка могут быть осложненным течением болезни Гиршпрунга.

У пациентов старшей возрастной группы клинические проявления болезни Гиршпрунга представляют собой упорные запоры, которые могут чередоваться с эпизодами энтероколита; увеличение размеров живота; отставание в физическом развитии.

Основные методы диагностики болезни Гиршпрунга включают ирригоскопию, аноректальную манометрию, ректальную полнослойную биопсию и биопсию слизистой прямой кишки для гистохимического или иммуногистохимического исследования.

Рентгенологическое исследование. На обзорной рентгенограмме у ребенка с болезнью Гиршпрунга можно найти избыточную пневмотизацию и расширение толстой кишки над узким дистальным участком прямой и сигмовидной кишок и переходной зоной между ними. При выраженном энтероколите будут признаки токсической дилятации толстой кишки $[10,11]$.

Для осложненной формы болезни Гиршпрунга в зависимости от клинической стадии будут характерные признаки низкой кишечной непроходимости или перфорации полого органа с наличием газа в свободной брюшной полости.

В некоторых центрах ирригоскопия является первым тестом в обследовании детей с подозрением на болезнь Гиршпрунга с признаками кишечной непроходимости. Признаками болезни Гиршпрунга на ирригоскопии являются патологическое сужение дистальных отделов толстой кишки, переходная зона в виде воронкообразного расширения над пораженным участком и проявления энтероколита, а также ненормальный ректосигмоидный индекс, который более выражен у детей старшей возрастной группы [12, 13]. Исследования последних лет показывают, что чувствительность и специфичность ирригоскопии у детей старшей возрастной группы составляет $70-83 \%$, в то время как у новорожденных пациентов этот процент значительно ниже из-за нечетко выраженной переходной зоны и отсутствия воронкообразного расширения кишки над ней. Кроме того, при наличии сопутствующего энтероколита раздраженная кишка с участками спазма может создавать видимость патологического сужения кишки. Это означает, что до месячного возраста пациента риск как ложно-положительного, так и ложно-отрицательного результата ирригоскопии увеличивается почти в три раза. Также, может быть несовпадение зоны рентгенологической и зоны, определенной при биопсии, особенно у детей до 1 месяца. Таким образом, ирригоскопия у новорожденных может быть неправильно интерпретирована $[7,8,14]$.

Использование аноректальной манометрии возможно у старших детей для диагностики болезни Гиршпрунга, но аноректальная манометрия у новорожденных достаточно спорна, технически трудна и недостоверна в большинстве случаев [11].

При морфологическом исследовании биоптатов используется несколько методик: световая микроскопия с окраской гематоксилин-эозином полнослойных биоптатов прямой кишки; гистохимическое и иммуногистохимическое исследование биоптатов слизистой прямой кишки. Ректальная полнослойная биопсия представлена отсутствием ганглионарных клеток при окраске гематоксилин-эозином. Гистохимическое исследование биоптатов слизистой прямой кишки показывает присутствие 
ацетилхолин-позитивных гипертрофических нервных волокон, подтверждающих болезнь Гиршпрунга. Наряду с гематоксилин-эозин ректальной биопсией, использование гистохимической реакции на ацетилхолинэстеразу сделало морфологическую диагностику более простой и надежной $[7,8,11]$. У новорожденного ребенка выполнение полнослойной биопсии представляет собой большие технические сложности, в то время как биопсия слизистой прямой кишки - технически простая манипуляция. Кроме того, в дистальной части анального канала отсутствие ганглионарных клеток может быть вариантом нормы. Некоторые другие методики используются для постановки диагноза болезни Гиршпрунга, такие как лактатдегидрогеназа, сукцинатдегидрогеназа, NADPH-диафораза гистохимическое определение ферментов. Гистохимическая реакция на ацетилхолинэстеразу для постановки диагноза при болезни Гиршпрунга у новорожденных имеет чувствительность $91 \%$, специфичность - $100 \%$, ложно-отрицательный результат получается в $8 \%$ случаев. У новорожденных, особенно недоношенных или функционально незрелых, может быть снижено количество нервных волокон в стенке кишки даже при наличии болезни Гиршпрунга, поэтому уменьшение количества АХЭ-положительных волокон у новорожденных может приводить к ложно-отрицательному результату. Несмотря на эти ограничения, ректальная биопсия более чувствительный и специфический метод, чем ирригоскопия и анальная манометрия вместе взятые, даже без использования дополнительных иммуногистохимических методик.

Иммуногистохимическое исследование важный морфологический метод, который использует специфические антиген-антитело реакции.

Основные иммуногистохимические методы прямая и непрямая иммунофлюоресценция или прямая и непрямая ферментная иммуногистохимическая реакция. Много иммуногистохимических маркеров и окрасок используется для исследования и клинической диагностики болезни Гиршпрунга и других заболеваний кишечника. Наиболее часто используют исследование на кальретинин - общий маркер [7, 15, 16].

Кальретинин - это Са-содержащий протеин, который играет важную роль в организации и функционировании энтеральной нервной системы. Са-содержащие протеины участвуют в физиологическом кальциевом гомеостазе. Ганглионарные клетки и их производные выделяют кальретинин из подслизистого и межмышечного сплетения кишки нормальной ганглионарной кишки в отличие от аганглионарного сегмента при болезни Гиршпрунга, в котором отсутствует кальретинин. Отсутствие окраски на кальретинин в нервных отростках также показывает отсутствие кальретинина в соответствующих нервных клетках, что можно использовать как диагностический тест в диагностике аганглионарного сегмента. Важным аспектом иммуногистохимического исследования является то, что метод позволяет обнаружить различные нарушения иннервации, т.к. окрашивает все имеющиеся клетки, даже незрелые и маленькие. Однако для выявления нервной гипертрофии необходимы другие маркеры и методы.

\section{Лечение}

Подтверждение диагноза болезни Гиршпрунга является показанием к оперативному лечению.

Успехи в выхаживании новорожденных, анестезии и реанимации за последние десятилетия сделали возможным для детских хирургов выполнение одноэтапной коррекции болезни Гиршпрунга при неосложненной форме и исключении тотального аганглиоза. В большинстве случаев диагноз ставится в неонатальном периоде, многие центры применяют одноэтапную коррекцию с потрясающими результатами. Основными противопоказаниями к первичному трансанальному низведению являются тяжелые жизненно-значимые пороки развития, тяжелый энтероколит, выраженная дилятация проксимальных отделов кишки и нарушения общесоматического состояния в целом.

При этом вмешательтве аганглионарная кишка низводится трансанально и резецируется примерно на 10 см выше уровня переходной зоны. Накладывается колоанальный анастомоз между ганглионарной кишкой и анусом $[17,18]$.

В случаях наличия длинной зоны аганглиоза может применяться лапароскопическая мобилизация толстой кишки и ее трансанальное эндоректальное низведение [19, 20].

Если имеется энтероколит, то сначала проводится интенсивное лечение, включающее коррекцию водно-электролитного баланса, очистительные клизмы [7, 8].

При осложненной форме болезни Гиршпрунга (кишечная непроходимость, некротический энтероколит с токсической дилятацией толстой кишки, перфорация терминального отдела тонкой кишки, слепой или других отделов толстой кишки) - выведение стомы со взятием биопсий на разных уровнях толстой кишки и ректальная биопсия. После подтверждения диагноза болезни Гиршпрунга, определения зоны аганглиоза кишечника определяют вид 
дальнейшего хирургического лечения [9].

Наиболее серьезный прогноз при интестинальной форме аганглиоза кишечника. Тонкокишечная высокая стома неизбежно вызовет синдром короткой кишки и сомнительные перспективы после радикальной операции [20].

\section{Результаты и осложнения}

Скрупулезная техника, адекватный гемостаз, хорошее кровоснабжение для предупреждения ишемии, недопустимость перекрута и натяжения кишки должны предотвратить осложнения при трансанальном эндоректальном низведении кишки.

Энтероколит - главная причина увеличения заболеваемости и смертности после радикального лечения болезни Гиршпрунга [7, 9]. Частота послеоперационного энтероколита, по данным разной литературы, варьирует от 5\% до $42 \%$, в зависимости от определения и методов диагностики энтероколита [7]. Несмотря на успехи в лечении детей с болезнью Гиршпрунга, патогенез энтероколита продолжает оставаться неизвестным. Предположительно, обструктивные механизмы приводят к кишечному стазу с размножением бактерий, повреждением бактериями слизистой с последующим местным и общим воспалительным ответом. Факторы риска энтероколита включают ранний возраст, стриктуры анастомоза и нарушения питания. Предполагалось, что более короткий мышечный рукав может снизить частоту энтероколита, снизив возможность спазма. Все эти находки позволили предположить, что кишечный стаз и незрелый иммунитет слизистой оболочки из-за раннего возраста могут способствовать возникновению энтероколита [17].

Образования рубцового стеноза после радикального низведения - другое серьезное осложнение. Частота стеноза варьирует от $0 \%$ до $35 \%$ в зависимости от определения понятия $[18,19]$. Факторами риска стеноза являются ишемия и несостоятельность анастомоза, а также циркулярный анастомоз. Кроме того, как говорилось выше, стеноз является высоким фактором риска послеоперационного энтероколита. Большая часть стенозов лечится консервативно дилятацией, и только некоторые требуют более агрессивной хирургической коррекции. Техника наложения косого анастомоза ануса может снизить формирование стеноза.

Несостоятельность колоанального анастомоза в виде перитонита, перианальной флегмоны или формирования параректального свища требует выведения стомы до купирования воспалительного процесса. Такие осложнения встречаются очень редко, так как анастомоз защищен стенками анального канала. Однако в случае ишемии в области анастомоза или гематомы со вторичным ее инфицированием, возможно формирование воспалительного очага. В случае восстановления целостности кишки и отсутствия деформаций в зоне анастомоза производится закрытие стомы путем наложения анастомоза конец-в-конец. Однако, если кишка окажется дискредитированной, необходимо повторное низведение кишки с резекцией пораженного участка кишки и закрытием стомы.

Частота стула обычно высока (5-10 раз в день) сразу после операции. С течением времени, через полгода после операции, достигает 1-4 раз в день. Запоры наступают через несколько недель или месяцев после операции и зависят от типа операции, с большей вероятностью возникновения после операций с оставлением аганглионарной части кишки (Дюамель, Рибейн). Запор возникает примерно у $8 \%$ детей, но частота их может достигать $20 \%[18,20$, 21]. Последние публикации сообщают о $37 \%$ детей с нарушением эвакуации стула после операций по поводу болезни Гиршпрунга [22, 23]. Функциональные формы запоров могут быть вылечены консервативными методами, такими как клизмы или слабительные. Кроме того, персистирующие запоры как следствие ахалазии сфинктера, формирования стриктуры, неполной резекции аганглионарной части кишки или дисганглионарной кишки могут потребовать активных действий, таких как повторная ирригоскопия, биопсия. Метод лечения зависит от результатов обследования и может включать активные дилятации, ботокс, миэктомию или ренизведение [6, 7, 18].

Недержание кала определяется обычно у детей старше 4 лет. Большая серия исследований указывает на отсутствие недержания кала после операции трансанального низведения. Некоторые авторы в то же время указывают на плохое держание кала после низведения из-за нарушения консистенции стула или жидкий стул после операции у детей с длинной зоной аганглиоза. 44\% детей вынуждены соблюдать диету после операции во избежание запора или недержания [21, 22, 23]. Другие авторы указывают, что некоторые аспекты держания и консистенции стула меняются с годами $[17,18]$.

Энурез встречается в 5-26\% случаев и объясняется ятрогенным повреждением тазовых нервов или невропатией [7]. Применение лапароскопии или трансанальное низведение призваны уменьшить количество ятрогенных повреждений.

Нарушения стула, включая запор и недер- 
жание, и энурез сильно влияют на послеоперационное качество жизни пациентов с болезнью Гиршпрунга. Наличие короткого участка толстой кишки после ее резекции при длинной зоне аганглиоза приведет к плохому качеству жизни, которое, однако, с годами изменится, когда пациент научится контролировать процесс дефекации и помогать своему состоянию различными методами (клизма, диета и пр.). Задачи хирурга - уменьшить эти осложнения путем развития тщательной оперативной техники, послеоперационного и длительного диспансерного наблюдения.

\section{Заключение}

В диагностике и лечении болезни Гиршпрунга достигнуты большие успехи. Однако функциональные результаты оставляют желать лучшего. Причинами этого являются сроки проведения хирургической коррекции порока, а также выбор метода оперативного лечения, подходящий для каждого конкретного ребенка.

Изучение литературы показало, что если вопросы патогенеза заболевания уже не считаются спорными, то внедрение новых методик обследования и хирургического лечения попрежнему остается сложным и неоднозначным.

Анализ литературных данных показал возможность решения этих проблем при выработке программы обследования, учете всех имеющихся осложнений и определении соответствующей тактики лечения.

\section{ЛИТЕРАТУРА}

1. Somme S, Langer JC. Primary versus staged pullthrough for the treatment of Hirschsprung disease. Semin Pediatr Surg. 2004 Nov;13(4):249-55.

2. Gariepy CE. Intestinal motility disorders and development of the enteric nervous system. Pediatr Res. 2001 May;49(5):605-13.

3. Tam PK, Garcia-Barcelo M. Molecular genetics of Hirschsprung's disease. Semin Pediatr Surg. 2004 Nov;13(4):236-48.

4. Amiel J, Sproat-Emison E, Garcia-Barcelo M, Lantieri F, Burzynski G, Borrego S, et al. Hirschsprung Disease Consortium. Hirschsprung disease, associated syndromes and genetics: a review. J Med Genet. 2008 Jan;45(1):1-14.

5. Kim JH, Yoon KO, Kim JK, Kim JW, Lee SK, Kong SY, et al. Novel mutations of RET gene in Korean patients with sporadic Hirschsprung's disease. $J$ Pediatr Surg. 2006 Jul;41(7):1250-54.

6. Swenson O. Hirschsprung's disease: a review. Pediatrics. 2002 May; 109(Is 5):914-18.

7. Holschneider AM, Puri P, eds. Hirschsprung's Disease and Allied Disorders. 3rd ed. New York, NY: Springer; 2008. 414 p.

8. Dasgupta R, Langer JC. Hirschsprung disease. Curr Probl Surg. 2004;41(12):942-88.
9. Yan Z, Poroyko V, Gu S, Zhang Z, Pan L, Wang $\mathbf{J}$, et al. Characterization of the intestinal microbiome of Hirschsprung's disease with and without enterocolitis. Biochem Biophys Res Commun. 2014 Mar 7;445(2):269-74. doi: 10.1016/j.bbrc.2014.01.104.

10. Diamond IR, Casadiego G, Traubici J, Langer JC, Wales PW. The contrast enema for Hirschsprung disease: predictors of a false-positive result. J Pediatr Surg. 2007 May;42(5):792-95.

11. De Lorijn F, Reitsma JB, Voskuijl WP, Aronson DC, Ten Kate FJ, Smets AM, et al. Diagnosis of Hirschsprung's disease: a prospective, comparative accuracy study of common tests. J Pediatr. 2005 Jun;146(6):787-92.

12. Garcia R, Arcement C, Hormaza L, Haymon ML, Ward K, Velasco C, et al. Use of the rectosigmoid index to diagnose Hirschsprung's disease. Clin Pediatr (Phila). 2007 Jan;46(1):59-63.

13. Proctor ML, Traubici J, Langer JC, Gibbs DL, Ein SH, Daneman A, et al. Correlation between radiographic transition zone and level of aganglionosis in Hirschsprung's disease: Implications for surgical approach. J Pediatr Surg. 2003 May;38(5):775-78.

14. Pratap A, Gupta DK, Tiwari A, Sinha AK, Bhatta N, Singh SN, et al. Application of a plain abdominal radiograph transition zone (PARTZ) in Hirschsprung's disease. BMC Pediatrics. 2007;7:5. doi: 10.1186/1471-2431-7-5.

15. De la Torre L, Santos k. Hirschsprung disease. Evaluation of calretinin and S-100 as ancillary methods for the diagnosis of aganglionosis in rectal biopsies. Acta Pediatr Mex. 2012 Sep-Oct;33(5):246-51.

16. Holland SK, Ramalingam P, Podolsky RH, ReidNicholson MD, Lee JR. Calretinin immunostaining as an adjunct in the diagnosis of Hirschsprung disease. Ann Diagn Pathol. 2011 Oct;15(5):323-28. doi: 10.1016/j. anndiagpath.2011.02.010.

17. Spitz L, Coran AG, eds. Operative Pediatric Surgery. 6th ed. London: Hodder Arnold; 2007. 1060 p. 18. Nasr A, Langer JC. Evolution of the technique in the transanal pull-through for Hirschsprung's disease: effect on outcome. J Pediatr Surg. 2007 Jan;42(1):36-9; discussion 39-40.

19. Georgeson KE, Robertson DJ. Laparoscopicassisted approaches for the definitive surgery for Hirschsprung's disease. Semin Pediatr Surg. 2004 Nov;13(4):256-62.

20. Cobellis G, Noviello C, Cruccetti A, Romano M, Mastroianni L, Amici G, et al. Staged laparoscopicassisted endorectal pull-through for long segment Hirschprung's disease and total colonic aganglionosis. Minerva Pediatr. 2011 Jun;63(3):163-67.

21. Bradnock TJ, Walker GM. Evolution in the management of Hirschsprung's disease in the UK and Ireland: a national survey of practice revisited. Ann $R$ Coll Surg Engl. 2011 Jan; 93(1): 34-38. doi: 10.1308/003588 410X12771863936846.

22. Huang EY, Tolley EA, Blakely ML, Langham MR. Changes in hospital utilization and management of Hirschsprung disease: analysis using the kids' inpatient database. Ann Surg. 2013 Feb;257(2):371-7. doi: 10.1097/SLA.0b013e31827ee976.

23. Niramis R, Watanatittan S, Anuntkosol M, Buranakijcharoen V, Rattanasuwan T, Tongsin A, et al. Quality of life of patients with Hirschsprung's disease at 5 - 20 years post pull-through operations. Eur $J$ Pediatr Surg. 2008 Feb;18(1):38-43. doi: 10.1055/s2008-1038325. 


\section{REFERENCES}

1. Somme S, Langer JC. Primary versus staged pullthrough for the treatment of Hirschsprung disease. Semin Pediatr Surg. 2004 Nov;13(4):249-55.

2. Gariepy CE. Intestinal motility disorders and development of the enteric nervous system. Pediatr Res. 2001 May;49(5):605-13.

3. Tam PK, Garcia-Barcelo M. Molecular genetics of Hirschsprung's disease. Semin Pediatr Surg. 2004 Nov;13(4):236-48.

4. Amiel J, Sproat-Emison E, Garcia-Barcelo M, Lantieri F, Burzynski G, Borrego S, et al. Hirschsprung Disease Consortium. Hirschsprung disease, associated syndromes and genetics: a review. J Med Genet. 2008 Jan;45(1):1-14. 5. Kim JH, Yoon KO, Kim JK, Kim JW, Lee SK, Kong SY, et al. Novel mutations of RET gene in Korean patients with sporadic Hirschsprung's disease. $J$ Pediatr Surg. 2006 Jul;41(7):1250-54.

6. Swenson O. Hirschsprung's disease: a review. Pediatrics. 2002 May; 109(Is 5):914-18.

7. Holschneider AM, Puri P, eds. Hirschsprung's Disease and Allied Disorders. 3rd ed. New York, NY: Springer; 2008. 414 p.

8. Dasgupta R, Langer JC. Hirschsprung disease. Curr Probl Surg. 2004;41(12):942-88.

9. Yan Z, Poroyko V, Gu S, Zhang Z, Pan L, Wang $\mathrm{J}$, et al. Characterization of the intestinal microbiome of Hirschsprung's disease with and without enterocolitis. Biochem Biophys Res Commun. 2014 Mar 7;445(2):269-74. doi: 10.1016/j.bbrc.2014.01.104.

10. Diamond IR, Casadiego G, Traubici J, Langer JC, Wales PW. The contrast enema for Hirschsprung disease: predictors of a false-positive result. J Pediatr Surg. 2007 May;42(5):792-95.

11. De Lorijn F, Reitsma JB, Voskuijl WP, Aronson DC, Ten Kate FJ, Smets AM, et al. Diagnosis of Hirschsprung's disease: a prospective, comparative accuracy study of common tests. J Pediatr. 2005 Jun;146(6):787-92.

12. Garcia R, Arcement C, Hormaza L, Haymon ML, Ward K, Velasco C, et al. Use of the recto-sigmoid indexto diagnose Hirschsprung's disease. Clin Pediatr (Phila). 2007 Jan;46(1):59-63.

13. Proctor ML, Traubici J, Langer JC, Gibbs DL, Ein SH, Daneman A, et al. Correlation between radiographic transition zone and level of aganglionosis

\section{Адрес для корреспонденции}

220013, Республика Беларусь,

г. Минск, пр. Независимости, д. 64,

ГУ «Республиканский научно-практический

центр детской хирургии»,

тел. моб.: +375 291696581 ,

e-mail: govorukhina@mail.ru,

Говорухина Ольга Алексеевна

\section{Сведения об авторах}

Говорухина О.А., к.м.н., доцент, заведующий хирургическим отделением №2 ГУ «Республиканский научно-практический центр детской хирургии».

\section{Информация о статье}

Поступила 5 ноября 2016 г.

Принята в печать 6 февраля 2017 г.

Доступна на сайте 25 сентября 2017 г. in Hirschsprung's disease: Implications for surgical approach. J Pediatr Surg. 2003 May;38(5):775-78.

14. Pratap A, Gupta DK, Tiwari A, Sinha AK, Bhatta N, Singh SN, et al. Application of a plain abdominal radiograph transition zone (PARTZ) in Hirschsprung's disease. BMC Pediatrics. 2007;7:5. doi: 10.1186/1471-2431-7-5.

15. De la Torre L, Santos k. Hirschsprung disease. Evaluation of calretinin and S-100 as ancillary methods for the diagnosis of aganglionosis in rectal biopsies. Acta Pediatr Mex. 2012 Sep-Oct;33(5):246-51.

16. Holland SK, Ramalingam P, Podolsky RH, ReidNicholson MD, Lee JR. Calretinin immunostaining as an adjunct in the diagnosis of Hirschsprung disease. Ann Diagn Pathol. 2011 Oct;15(5):323-28. doi: 10.1016/j. anndiagpath.2011.02.010.

17. Spitz L, Coran AG, eds. Operative Pediatric Surgery. 6th ed. London: Hodder Arnold; 2007. 1060 p. 18. Nasr A, Langer JC. Evolution of the technique in the transanal pull-through for Hirschsprung's disease: effect on outcome. J Pediatr Surg. 2007 Jan;42(1):36-9; discussion 39-40.

19. Georgeson KE, Robertson DJ. Laparoscopic-assisted approaches for the definitive surgery for Hirschsprung's disease. Semin Pediatr Surg. 2004 Nov;13(4):256-62.

20. Cobellis G, Noviello C, Cruccetti A, Romano M, Mastroianni L, Amici G, et al. Staged laparoscopicassisted endorectal pull-through for long segment Hirschprung's disease and total colonic aganglionosis. Minerva Pediatr. 2011 Jun;63(3):163-67.

21. Bradnock TJ, Walker GM. Evolution in the management of Hirschsprung's disease in the UK and Ireland: a national survey of practice revisited. Ann $R$ Coll Surg Engl. 2011 Jan; 93(1): 34-38. doi: 10.1308/003588 410X12771863936846.

22. Huang EY, Tolley EA, Blakely ML, Langham MR. Changes in hospital utilization and management of Hirschsprung disease: analysis using the kids' inpatient database. Ann Surg. 2013 Feb;257(2):371-7. doi: 10.1097/SLA.0b013e31827ee976.

23. Niramis R, Watanatittan S, Anuntkosol M, Buranakijcharoen V, Rattanasuwan T, Tongsin A, et al. Quality of life of patients with Hirschsprung's disease at $5-20$ years post pull-through operations. Eur J Pediatr Surg. 2008 Feb;18(1):38-43. doi: 10.1055/s2008-1038325.

\section{Address for correspondence}

220013, Republic of Belarus,

Minsk, Nezavisimost Ave., 64,

SI «Republican Scientific

and Practical Center for Pediatric Surgery»,

Tel.: +37529 1696581,

E-mail: govorukhina@mail.ru

Olga A. Govorukhina

\section{Information about the authors}

Govorukhina O.A. PhD, Ass. Professor, Head of Department of Surgical Diseases N 2, SE "Republican Scientific and Practical Center of Pediatric Surgery".

\section{Article history}

Arrived 5 November 2016

Accepted for publication 6 February 2017

Available online 25 September 2017 